\title{
Associations between body mass index and bladder cancer survival: Is the obesity paradox short-lived?
}

Fernanda Arthuso; Adrian S. Fairey; Normand G. Boule; Kerry S. Courneya

University of Alberta, Edmonton, AB, Canada

Cite as: Arthuso F, Fairey AS, Boule NG, et al. Associations between body mass index and bladder cancer survival: Is the obesity paradox short-lived? Can Urol Assoc J 2021 December 21; Epub ahead of print. http://dx.doi.org/10.5489/cuaj.7546

Published online December 21, 2021

Corresponding author: Dr. Fernanda Arthuso, University of Alberta, Edmonton, AB, Canada; arthuso@ualberta.ca

$* * *$

\section{Abstract}

Introduction: We investigated the associations of pre-surgical body mass index (BMI) with bladder cancer outcomes in patients treated with radical cystectomy.

Methods: We retrospectively analyzed data from 488 bladder cancer patients treated with radical cystectomy between 1994 and 2007 and followed up until 2016. Cox regression with step function (time-segment analysis) was conducted for overall survival because the proportional hazard assumption was violated.

Results: Of 488 bladder cancer patients, 155 (31.8\%) were normal weight, 186 (38.1\%) were overweight, and 147 (30.1\%) were obese. During the median followup of 59.5 months, 363 (74.4\%) patients died, including 197 (40.4\%) from bladder cancer. In adjusted Cox regression analyses, BMI was not significantly associated with bladder cancer-specific survival for overweight (hazard ratio [HR] 0.79, 95\% confidence interval [CI] 0.57-1.10, $\mathrm{p}=0.16$ ) or obese (HR $0.76,95 \%$ CI $0.52-1.09, \mathrm{p}=0.13$ ) patients. In the Cox regression with step function for overall survival, the time interaction was significant overall $(\mathrm{p}=0.020)$ and specifically for overweight patients $(\mathrm{p}=0.006)$. In the time-segment model, the HR for overweight during the first 63 months was $0.66(95 \%$ CI $0.49-0.90, p=0.008)$, whereas it was 1.41 (95\% CI $0.89-2.23$, $\mathrm{p}=0.14$ ) after 63 months. Although not statistically significant, a similar pattern was observed for obese patients.

Conclusions: Our findings suggest that overweight and obese bladder cancer patients had better outcomes within the first five years after radical cystectomy; however, there were no differences 
in longer-term survival. These data suggest that the obesity paradox in bladder cancer patients treated with radical cystectomy may be short-lived.

\section{Introduction}

Bladder cancer is the fifth most common cancer in Canada ${ }^{1}$ and the tenth most common cancer worldwide. ${ }^{2}$ Radical cystectomy is the main treatment for muscle invasive bladder cancer and high risk non-muscle invasive bladder cancer ${ }^{3}$. Although bladder cancer survival has increased slightly, ${ }^{4}$ bladder cancer remains the eighth most common cause of cancer mortality in Canada. ${ }^{1}$ The 90-day mortality rate after radical cystectomy ranges from $3.2 \%-7.5 \%{ }^{5,6}$ and the five-year relative survival rate ranges from $16 \%$ to $55 \%$ depending on the tumor stage. ${ }^{7}$

Overweight and obesity are potential risk factors for developing bladder cancer because fat mass is related to inflammatory processes, alterations in sex hormone metabolism, abnormal levels of insulin and insulin-like growth factor, adipokine pathways, and microenvironment perturbations that contribute to tumor cell growth and proliferation. ${ }^{8,9}$ The association of obesity with bladder cancer survival, however, is much less clear. Some studies have reported that higher body mass index (BMI) is associated with a worse prognosis ${ }^{10,11}$ whereas other studies have found no association. ${ }^{12,13}$ Conversely, some studies have reported that higher BMI is associated with better survival in bladder cancer patients, ${ }^{14-16}$ a phenomenon known as the obesity paradox.

Obesity may be linked to cancer treatment-related symptoms, treatment tolerance and response, recurrence, and long-term survival. Understanding the association between obesity and survival in bladder cancer patients after radical cystectomy may improve prognosis and guide bladder cancer management. The purpose of the present study was to investigate the association of pre-surgical BMI with bladder cancer outcomes in patients treated with radical cystectomy.

\section{Methods}

After institutional review board approval (Health Research Ethics Board of Alberta (HREBA) Cancer Committee \#20-0294), we analyzed an existing database of bladder cancer patients undergoing radical cystectomy from the Northern Alberta Urology Centre in Edmonton, Canada. The data set was collected retrospectively and included bladder cancer patients undergoing radical cystectomy between 1994 and 2007 and followed-up for mortality outcomes until 2016. The primary exposure of interest was BMI defined as weight in kilograms divided by the square of height in meters $\left(\mathrm{kg} / \mathrm{m}^{2}\right)$. BMI was measured objectively during a clinical visit prior to radical cystectomy and was recorded in the patient electronic medical record. Eligible patients had to have a measure of BMI recorded in the data set.

The primary outcomes were length of hospitalization, 90-day mortality, bladder cancerspecific survival (BCSS), and overall survival (OS). Length of hospitalization was the number of days hospitalized from the time of radical cystectomy until discharge. 90-day mortality was assessed as death from any cause within 90 days of the radical cystectomy. BCSS was defined as 
the time from radical cystectomy to the date of death from bladder cancer or last follow-up if the patient had not died of bladder cancer. If the patient died of other causes, the event was censored at the time of death. OS was determined as the time from radical cystectomy to the date of death from any cause. Patients who were still alive were censored at the date of last follow-up.

Patients were categorized as normal weight (18.5-24.9 kg/m²), overweight (25-29.9 $\left.\mathrm{kg} / \mathrm{m}^{2}\right)$, or obese $\left(\geq 30 \mathrm{~kg} / \mathrm{m}^{2}\right)$. Descriptive statistics were used to summarize the data overall and by BMI category. Missing values for covariates $(<3 \%)$ were replaced with the mean for continuous variables and the mode for categorical variables. Univariable associations between BMI and clinicopathologic variables were assessed using Pearson chi-square for categorical variables and ANOVA for continuous variables. Life tables were used to obtain median survival time overall and by BMI category. Kaplan-Meier curves were used to describe survival probabilities of OS by BMI category. Log-rank tests were used to assess differences in unadjusted survival probabilities by BMI category.

Proportional hazard assumption was verified graphically using the log minus log plot and considered valid if the curves run in parallel. Cox proportional model was used to estimate the hazard ratio (HR) and 95\% confidence intervals (CI) for 90-day mortality and BCSS. We observed evidence of non-proportionality for OS, indicating that the effect of a fixed baseline covariate (i.e. BMI) may change over time. ${ }^{17}$ Therefore, a Cox regression with a step-function (time-segment analysis) was applied to explore the time varying coefficient. ${ }^{17,18}$ Two separate analyses were conducted based on a split of the median survival time. ${ }^{18}$ All the survival analyses were adjusted for established prognostic factors including age, sex, cancer subtype (urothelial vs non-urothelial), lymphovascular invasion, tumor grade, pathologic $\mathrm{T}$ stage, pathologic $\mathrm{N}$ stage, and adjuvant chemotherapy. All tests were 2 -sided and statistical significance was set at $\mathrm{p}<0.05$. All analyses were performed using SPSS (SPSS Inc., Chicago, IL, USA).

\section{Results}

A total of 508 patients were initially available in the data set. Twenty patients were excluded from the analyses due to missing data on weight or height $(\mathrm{n}=6)$, implausible BMI $(\mathrm{n}=4)$, and being underweight $(\mathrm{n}=10)$. The final sample included 488 bladder cancer patients who underwent radical cystectomy and had a valid measure of BMI before surgery.

Table 1 summarizes the baseline demographic and disease characteristics of the bladder cancer patients overall and by BMI category. Mean age at diagnosis was 65.8 years, BMI was $27.7 \mathrm{~kg} / \mathrm{m}^{2}$, and $78.5 \%$ were males. Most patients were diagnosed with urothelial carcinoma (89.8\%), high-grade tumors (88.5\%), and $\geq \mathrm{T} 2$ stage $(71.1 \%)$. Overall, 155 patients (31.8\%) were normal weight, 186 (38.1\%) were overweight, and 147 (30.1\%) were obese. Sex differed among the BMI categories, with the overweight and obese groups having more men $(\mathrm{p}<0.05)$. Regarding disease profile, overweight and obese patients generally had a more favorable prognostic profile compared to normal weight patients including a significantly lower pathologic $\mathrm{T}$ stage $(\mathrm{p}<0.05)$. 
Table 2 summarizes the treatment and survival outcomes of bladder cancer patients overall and by BMI category. Only $1 \%$ of patients underwent neoadjuvant chemotherapy. Surgical procedures did not vary among BMI categories; all women in the study underwent anterior exenteration and $98.2 \%$ of men had a radical cystoprostatectomy; $85.2 \%$ of patients had a standard lymph node dissection; and $77.7 \%$ had ileal conduit diversion. In the 30 -day mortality analyses, overweight patients had a lower proportion of deaths compared to normal and obese patients $(\mathrm{p}=0.039)$ but the overall number of deaths was small $(\mathrm{n}=15 ; 3.1 \%)$.

Regarding our primary outcomes of interest, obese patients had a prolonged hospital stay compared to overweight patients $(17.5 \pm 1.4$ vs $13.2 \pm 1.3$ days, $\mathrm{p}<0.05)$. After adjusting for prognostic factors, hospitalization remained significantly longer for obese versus overweight $(17.8 \pm 1.4$ vs $13.0 \pm 1.2$ days; $\mathrm{p}=0.010)$ patients and became significantly longer for obese versus normal weight $(17.8 \pm 1.4$ vs $14.0 \pm 1.4$ days; $p=0.036)$ patients. In the 90 -day mortality analyses, overweight patients had a lower proportion of deaths compared to normal and obese patients $(\mathrm{p}<0.001)$. The results remained statistically significant in the multivariable Cox regression analyses where overweight patients ( $\mathrm{HR}=0.09 ; 95 \% \mathrm{CI}$ : $0.02-0.38 ; \mathrm{p}=0.001)$ were less likely to die within 90-days after radical cystectomy (Table 3).

During the median follow-up of 59.5 months (interquartile range, 13-144), 363 (74.4\%) patients died including 197 (40.4\%) from bladder cancer. Univariable analyses for bladder cancer-specific mortality showed that being obese was associated with improved BCSS $(p=0.011)$. After adjusting for established prognostic factors in the multivariable Cox regression analyses (Table 3), the difference in BCSS was not significant for either overweight $(\mathrm{HR}=0.79$; 95\% CI: 0.57 to $1.10 ; p=0.16)$ or obese $(\mathrm{HR}=0.76 ; 95 \% \mathrm{CI}: 0.52$ to $1.09 ; \mathrm{p}=0.13$ ) patients. The supplementary table provides the multivariate analysis including all the covariates for the association of BMI with 90-day mortality, bladder cancer-specific mortality, and all-cause mortality.

Median OS for normal weight, overweight, and obese patients was 34.4, 88.5, and 70.8 months, respectively. When assessing the proportional hazard assumption using the graphical model log-log plot, we observed evidence of non-proportional hazards among the BMI categories for OS. The violation of the proportional hazard assumption indicates that the hazard is transient over time (i.e. time-varying coefficient) and may lead to biased effect estimates when follow-up is long. One method to address the non-proportionality is dividing the Cox model into time-intervals, therefore, we conducted two separate analyses split at the median survival of 63 months. In this analysis, BMI was included as a time-varying coefficient and the overall timeinteraction was significant $(\mathrm{p}=0.020)$. More specifically, the interaction was significant for overweight $(\mathrm{HR}=2.13$; 95\% CI: $1.24-3.66 ; \mathrm{p}=0.006)$ but not obese, although the interaction was in the same direction $(\mathrm{HR}=1.57 ; 95 \% \mathrm{CI}: 0.87-2.82 ; \mathrm{p}=0.13)$. In the time-segment model, the estimated HR for overweight during the first 63 months was 0.66 (95\% CI: 0.49-0.90, p=0.008) whereas it was 1.41 (95\% CI: 0.89-2.23, p=0.14) after 63 months. The estimated hazards ratio 
for obese during the first 63 months was 0.88 ( $95 \%$ CI: $0.64-1.20, \mathrm{p}=0.42$ ) whereas it was 1.38 ( $95 \%$ CI: $0.84-2.28, \mathrm{p}=0.21$ ) after 63 months (Table 3 ). We conducted a sensitivity analysis for all survival analyses that excluded non-urothelial cancers and found no meaningful differences compared to the overall analyses (data not shown).

\section{Discussion}

In this retrospective analysis of a previously established database, overweight and obese patients had a lower 90-day mortality rate and a better OS within the first five years of radical cystectomy. After five years, however, overweight and obese patients had an increased risk of all-cause mortality, resulting in no difference in longer-term OS. These data suggest that the obesity paradox in bladder cancer patients treated with radical cystectomy may be driven by a lower 90-day mortality rate and fewer early bladder cancer-specific deaths and, therefore, shortlived. Our findings suggest a more nuanced interpretation of the obesity paradox for bladder cancer survival.

The role of BMI as a predictor of survival outcomes in bladder cancer patients varies considerably among studies. ${ }^{19,20}$ A recent systematic review and meta-analysis of BMI and survival in urothelial cancer patients treated with radical surgery reported that overweight was associated with better BCSS (HR $=0.793 ; 95 \%$ CI:0.706-0.891; $\mathrm{p}<0.001)$ but worse OS $(\mathrm{HR}=1.125 ; 95 \% \mathrm{CI}: 1.018-1.244 ; \mathrm{p}=0.021)$. Conversely, obesity was significantly associated with both worse BCSS (HR=1.138; 95\% CI:1.028-1.261; $\mathrm{p}=0.013$ ) and OS (HR=1.308; 95\% CI:1.192-1.436; $\mathrm{p}<0.001) .{ }^{20}$ This review noted substantial heterogeneity among the studies, although the source of the variance was not identified. One possible source of heterogeneity is that the review included Asian and Western-based studies with varying definitions of the BMI categories. Moreover, the studies had relatively short median follow-up times (from 21 to 64.1 months) making it difficult to compare the short-term and long-term survival experience of overweight and obese bladder cancer patients.

The obesity paradox appears to only hold during the first five years after radical cystectomy, when most deaths are due to surgical complications or bladder cancer, and then dissipates during further follow-up when most deaths are due to other causes. After five years, obesity appears to exert its usual negative association with survival. This unique finding in our study was prompted by a violation of the proportional hazard assumption in our data set, which indicated BMI as a time-varying coefficient for OS. ${ }^{21}$ Assessment of the proportionality of hazards is a fundamental step for survival analyses using the Cox model. When the assumption is violated, a significant effect in the early (or late) follow-up period may be missed. ${ }^{18}$ The verification of this assumption, however, is not commonly reported in survival analysis of BMI in oncology, including investigations in bladder cancer. ${ }^{11-15,22}$

It is possible that our observation may provide another explanation of the inconsistency in the obesity paradox across different cancers. Petrelli et al. ${ }^{23}$ observed that the association between obesity and cancer survival may differ based on cancer type. More specifically, these 
authors reported evidence of the obesity paradox in lung, renal, and metastatic melanoma cancers; all cancers that generally have higher rates of surgical complications and more cancer deaths that occur early in the cancer trajectory. Conversely, they noted that the traditional negative association of BMI with survival seemed to hold in patients with breast, colon, and uterine cancers; all cancers that generally have lower rates of surgical complications and fewer early cancer-specific deaths, where patients are more likely to die of other causes during longerterm follow-up.

Nevertheless, the obesity paradox, whether short-lived or not, should be interpreted with caution. Explanations in the literature include methodological biases, such as unmeasured confounding. ${ }^{24}$ For instance, smoking status was not assessed in our study and it may confound the association between BMI and survival in bladder cancer patients. Other studies have suggested clinical and biological differences among BMI categories including body composition and tumor expression. ${ }^{24}$

For example, in patients with clear cell renal cell carcinoma, another malignancy of the genitourinary tract, differences in gene expression pathways within the tumor microenvironment might be associated with the obesity paradox. ${ }^{25,26}$ Sanchez et al. ${ }^{25}$ reported that obese patients had significant tumor up-regulation angiogenic pathways in comparison to normal weight patients; therefore they were more likely to increase local drug delivery and benefit from therapy. Hakimi et al. ${ }^{26}$ identified that fatty acid synthase (FASN) gene expression, an indicator of tumor aggressiveness and prognosis, differed by BMI categories. FASN was significantly upregulated in the normal BMI group and downregulated in the obese group, suggesting that the tumors of obese patients may be less aggressive than normal weight patients with renal cell carcinoma. ${ }^{26}$ In our sample, overweight and obese patients had a more favorable prognostic profile compared to normal weight patients. The literature has conflicting findings, however, regarding the associations of BMI with tumor characteristics in bladder cancer patients and further investigation is needed. ${ }^{10,22}$

BMI as a measure of obesity is also questionable since it fails to distinguish body composition compartments among individuals in the same BMI range. ${ }^{27}$ Psutka et al. ${ }^{16}$ explored the association of BMI and fat mass index with OS in patients treated with radical cystectomy and urinary diversion. Increased BMI was associated with improved 5-year OS, however, obesity based on fat-mass index had no association. In addition, after adjusting for the presence of low skeletal muscle mass, the association disappeared. ${ }^{16}$ Skeletal muscle mass is an important prognostic factor not assessed by BMI. Bladder cancer patients with lower skeletal muscle mass are at increased risk of early complications, cancer specific mortality, and all-cause mortality. ${ }^{28,}$ ${ }^{29}$ Bladder cancer patients have a high prevalence of low skeletal muscle mass at diagnosis and post-treatment. ${ }^{30}$ It is plausible that patients in the normal weight range might have lower muscle mass and, therefore, lower survival rates compared to overweight and obese patients. Another possible explanation for better survival rates among overweight patients relates to the 
amount of subcutaneous fat. Cancer patients with higher subcutaneous adipose tissue experience longer survival than those with low subcutaneous fat. ${ }^{31}$ The moderate amount of fat allows patients to survive weight losses that occur with treatment and may provide nutritional benefits. 24, 32 These explanations are only speculations, however, and further research is warranted.

Our study has important strengths and limitations. The strengths of our study include a sufficient sample size, the balanced distribution across the BMI categories, the relatively long follow-up time, and the verification of the proportional hazard assumption for the survival analysis. The limitations of our study include the exploratory nature of the analysis, the retrospective design of the study, the lack of data on potential confounders (e.g., smoking status, comorbidities), the use of BMI as the measure of obesity, and the measurement of BMI at only one time point. In addition, treatment has evolved since the completion of data collection (19942007) which may alter the association of obesity with survival. For example, our 90 -day mortality rate of $12.3 \%$ is higher than that reported in the current literature, possibly due to changes in treatments and/or the likely higher rate of normal weight patients in our more dated sample. Finally, the generalizability of our results is limited only to patients treated with radical cystectomy. Future research should collect more sophisticated measures of obesity at multiple time points and check the proportional hazard assumption for a potential time-varying interaction with obesity. Moreover, other measures of health-related fitness besides body composition (e.g., cardiovascular fitness, muscular strength, physical functioning) may also be important predictors of clinical outcomes in bladder cancer patients as they have been in breast cancer patients. ${ }^{33}$

\section{Conclusions}

This exploratory analysis suggests that the obesity paradox in bladder cancer patients treated with radical cystectomy may only apply to postsurgical complications and bladder cancer deaths that typically occur early in the survival trajectory. With sufficient follow-up, the benefits of obesity on bladder cancer survival are reversed by an increased risk of other deaths, resulting in no long-term survival advantage for overweight/obese bladder cancer patients. Whether these associations were due to unmeasured confounding, limitations of BMI, or different tumor expression among the BMI groups is unknown. Well designed, prospective studies that include a direct measure of body composition and tumor expression pathways are warranted to understand if and how body composition is associated with survival outcomes in bladder cancer. 


\section{References}

1. Brenner DR, Weir HK, Demers AA et al. Projected estimates of cancer in Canada in 2020. CMAJ 2020;192:E199-205.

2. International Agency for Research on Cancer. Estimated number of incident cases worldwide, both sexes, all ages. http://gco.iarc.fr/today/home Accessed July 28, 2021

3. National Comprehensive Cancer Network. NCCN Clinical Practice Guidelines on Oncology: Bladder Cancer, 2021

4. Antoni S, Ferlay J, Soerjomataram I et al. Bladder cancer incidence and mortality: A global overview and recent trends. Eur Urol 2017; 71:96-108.

5. Zakaria AS, Santos F, Dragomir A. et al. Postoperative mortality and complications after radical cystectomy for bladder cancer in Quebec: A population-based analysis during the years 2000-2009. Can Urol Assoc J 2014; 8:259-67.

6. Yafi FA, Aprikian AG, Chin JL et al. Contemporary outcomes of 2287 patients with bladder cancer who were treated with radical cystectomy: a Canadian multicentre experience. BJU Int 2011; 108:539-45.

7. Surveillance \& Reporting. The 2019 Report on Cancer Statistics in Alberta Edmonton: CancerControl Alberta,, Alberta Health Services; 2019. https://public.tableau.com/profile/cancercontrol.ab\#!/vizhome/The2019ReportonCancer StatisticsinAlberta/Highlights?publish=yes. Accessed September 7, 2020

8. Sun JW, Zhao LG, Yang Y et al. Obesity and risk of bladder cancer: a dose-response meta-analysis of 15 cohort studies. PLoS One 2015; 10:e0119313.

9 Avgerinos KI, Spyrou N, Mantzoros CS. et al.: Obesity and cancer risk: Emerging biological mechanisms and perspectives. Metabolism, 92: 121, 2019

10. Chromecki TF, Cha EK, Fajkovic H. et al. Obesity is associated with worse oncological outcomes in patients treated with radical cystectomy. BJU Int 2013; 111: 249-55.

11. Dabi Y, Rouscoff Y, Anract J et al.: Impact of body mass index on the oncological outcomes of patients treated with radical cystectomy for muscle-invasive bladder cancer. World J Urol 2017; 35:229-35.

12. Hafron J, Mitra N, Dalbagni $G$ et al. Does body mass index affect survival of patients undergoing radical or partial cystectomy for bladder cancer? J Urol 2005; 173:1513-7.

13. Bachir BG, Aprikian AG, Izawa JI et al. Effect of body mass index on the outcomes of patients with upper and lower urinary tract cancers treated by radical surgery: results from a Canadian multicenter collaboration. Urol Oncol 2014; 32: 441-8.

14. Gierth M, Zeman F, Denzinger S et al. Influence of body mass index on clinical outcome parameters, complication rate and survival after radical cystectomy: Evidence from a prospective European multicentre study. Urol Int 2018; 101:16-24.

15. Bi H, Huang Y, Wang G et al. Impact of body mass index and pretreatment hemoglobin level on prognosis following radical cystectomy for bladder cancer in males and females. Urol Int 2020; 104: 28-35.

16. Psutka SP, Boorjian SA, Moynagh MR et al. Mortality after radical cystectomy: impact of obesity versus adiposity after adjusting for skeletal muscle wasting. J Urol 2015; 193:1507-1513. 
17. Zhang Z, Reinikainen J, Adeleke KA et al. Time-varying covariates and coefficients in Cox regression models. Ann Transl Med 2018; 6:121.

18. Schemper M. Cox analysis of survival data with non-proportional hazard functions. The Statistician 1992; 41:455-65.

19. Ornaghi PI, Afferi L, Antonelli A et al. The impact of preoperative nutritional status on post-surgical complication and mortality rates in patients undergoing radical cystectomy for bladder cancer: a systematic review of the literature. World J Urol 2020; 39:104581.

20. Yang Z, Bai Y, Hu X et al. The prognostic value of body mass index in patients with urothelial carcinoma after surgery: A systematic review and meta-analysis. Dose Response 2020; 181559325820979247, 2020

21. Lee L, Shih JH. An introduction to survival analysis. In: Principles and practice of clinical research, 2018, 373-381.

22. Kwon T, Jeong IG, You D et al. Obesity and prognosis in muscle-invasive bladder cancer: the continuing controversy. Int J Urol 2014, 21:1106-12.

23. Petrelli F, Cortellini A, Indini A et al. Association of obesity with survival outcomes in patients with cancer: A systematic review and meta-analysis. JAMA Netw Open 2021, 4: e213520.

24. Caan BJ, Cespedes Feliciano EM, Kroenke CH. The importance of body composition in explaining the overweight paradox in cancer-counterpoint. Cancer Res 2018; 78:190612.

25. Sanchez A, Furberg H, Kuo F et al. Transcriptomic signatures related to the obesity paradox in patients with clear cell renal cell carcinoma: a cohort study. Lancet Oncol 2020, 21:283-293.

26. Hakimi AA, Furberg H, Zabor EC et al. An epidemiologic and genomic investigation into the obesity paradox in renal cell carcinoma. J Natl Cancer Inst 2013; 105:1862-70

27. Sanchez A, Kissel S, Coletta A et al. Impact of body size and body composition on bladder cancer outcomes: Risk stratification and opportunity for novel interventions. Urol Oncol 2020; 38:713-18.

28. Mayr R, Gierth M, Zeman F et al. Sarcopenia as a comorbidity-independent predictor of survival following radical cystectomy for bladder cancer. J Cachexia Sarcopenia Muscle 2018; 9:505-13

29. Mayr R, Fritsche HM, Zeman F et al. Sarcopenia predicts 90-day mortality and postoperative complications after radical cystectomy for bladder cancer. World J Urol 2018; 36:1201-07

30. Hansen TTD, Omland LH, von Heymann A et al. Development of sarcopenia in patients with bladder cancer: A systematic review. Semin Oncol Nurs 2021; 37:151108

31. Ebadi M, Martin L, Ghosh S et al. Subcutaneous adiposity is an independent predictor of mortality in cancer patients. Br J Cancer 2017; 117:148-55.

32. Caan BJ, Meyerhardt JA, Kroenke $\mathrm{CH}$ et al. Explaining the obesity paradox: The association between body composition and colorectal cancer survival (C-SCANS Study). Cancer Epidemiol Biomarkers Prev 2017; 26:1008-15 
33. An KY, Arthuso FZ, Kang DW et al. Exercise and health-related fitness predictors of chemotherapy completion in breast cancer patients: pooled analysis of two multicenter trials. Breast Cancer Res Treat 2021, 188: 399-407 


\section{Figures and Tables}

\begin{tabular}{|c|c|c|c|c|c|}
\hline \multirow[b]{2}{*}{ Variables } & \multirow[b]{2}{*}{ Overall } & \multicolumn{3}{|c|}{ Body mass index $\left(\mathrm{kg} / \mathrm{m}^{2}\right)$} & \multirow[b]{2}{*}{$\mathbf{p}$} \\
\hline & & $\begin{array}{l}\text { Normal } \\
\text { weight } \\
18.5-24.9\end{array}$ & \begin{tabular}{|l|} 
Overweight \\
$25-29.9$ \\
\end{tabular} & $\begin{array}{l}\text { Obese } \\
\geq 30\end{array}$ & \\
\hline & $(\mathrm{n}=488)$ & $(n=155)$ & $(n=186)$ & $(\mathrm{n}=147)$ & \\
\hline $\begin{array}{l}\text { Body mass index }\left(\mathrm{kg} / \mathrm{m}^{2}\right) \\
\mathrm{M} \pm \mathrm{SD}\end{array}$ & $27.7 \pm 4.8$ & $22.6 \pm 1.6$ & $27.3 \pm 1.4$ & $33.4 \pm 3.3$ & - \\
\hline Weight (kg), $\mathrm{M} \pm \mathrm{SD}$ & $81.8 \pm 17.1$ & $65.8 \pm 9.5$ & $81.4 \pm 9.6$ & $99.3 \pm 13.5$ & - \\
\hline Sex (male), n (\%) & $383(78.5)$ & $107(69.0)$ & $154(82.8)^{\mathrm{a}}$ & $122(83.0)^{\mathrm{a}}$ & 0.002 \\
\hline Age (years), $\mathrm{M} \pm \mathrm{SD}$ & $65.8 \pm 10.0$ & $65.6 \pm 10.9$ & $66.7 \pm 9.9$ & $64.7 \pm 9.1$ & 0.18 \\
\hline Urothelial carcinoma, $\mathrm{n}(\%)$ & $438(89.8)$ & $138(89.0)$ & $164(88.2)$ & $136(92.5)$ & 0.41 \\
\hline Lymphovascular invasion, $\mathrm{n}(\%)$ & $153(31.4)$ & $59(38.1)$ & $55(29.6)$ & $39(26.5)^{a}$ & 0.08 \\
\hline High-grade, n (\%) & $432(88.5)$ & $143(92.3)$ & $159(85.5)$ & $130(88.4)$ & 0.15 \\
\hline Pathologic T stage ( $\geq \mathrm{T} 2), \mathrm{n}(\%)$ & $347(71.1)$ & $124(80.0)$ & $130(69.9)^{\mathrm{a}}$ & $93(63.3)^{\mathrm{a}}$ & 0.005 \\
\hline Pathologic $\mathrm{N}$ stage $(\mathrm{N}+), \mathrm{n}(\%)$ & $104(21.3)$ & $41(26.5)$ & $37(19.9)$ & $26(17.7)$ & 0.15 \\
\hline
\end{tabular}

${ }^{\mathrm{a}} \mathrm{p}<0.05$ vs. normal weight. M: mean; SD: standard deviation. 


\begin{tabular}{|c|c|c|c|c|c|}
\hline \multirow[b]{2}{*}{ Variables } & \multirow[b]{2}{*}{ Overall } & \multicolumn{3}{|c|}{ Body mass index $\left(\mathrm{kg} / \mathrm{m}^{2}\right)$} & \multirow[b]{2}{*}{$\mathbf{p}$} \\
\hline & & $\begin{array}{l}\text { Normal } \\
\text { weight } \\
18.5-24.9\end{array}$ & $\begin{array}{l}\text { Overweight } \\
25-29.9\end{array}$ & $\begin{array}{l}\text { Obese } \\
\geq 30\end{array}$ & \\
\hline & $(n=488)$ & $(\mathrm{n}=155)$ & $(n=186)$ & $(\mathrm{n}=147)$ & \\
\hline Neoadjuvant chemotherapy, n (\%) & $5(1.0)$ & $2(1.3)$ & $2(1.1)$ & $1(0.7)$ & \\
\hline \multicolumn{6}{|l|}{ Surgical procedure, $\mathrm{n}(\%)$} \\
\hline Anterior exenteration (women) & $105(100)$ & $48(100)$ & $32(100)$ & $25(100)$ & \\
\hline Radical cystoprostate (men) & $376(98.2)$ & $103(96.3)$ & $152(98.7)$ & $121(99.2)$ & \\
\hline Positive surgical margin, n (\%) & $60(12.3)$ & $18(11.6)$ & $23(12.4)$ & $19(12.9)$ & 0.94 \\
\hline Lymph node dissection, n (\%) & & & + & & \\
\hline None/limited & $37(7.6)$ & $18(11.6)$ & $12(6.5)$ & $7(4.8)$ & 0.63 \\
\hline Standard & $416(85.2)$ & $124(80.0)$ & $161(86.6)$ & $131(89.1)$ & \\
\hline Extended & $35(7.2)$ & $13(8.4)$ & $13(7.0)$ & $9(6.1)$ & \\
\hline \multicolumn{6}{|l|}{ Diversion, $\mathrm{n}(\%)$} \\
\hline Ileal conduit & $379(77.7)$ & $121(78.1)$ & $147(79.0)$ & $111(75.5)$ & 0.62 \\
\hline Neobladder & $99(20.3)$ & $29(18.7)$ & $36(19.4)$ & $34(23.1)$ & \\
\hline Other & $10(2.0)$ & $5(3.2)$ & $3(1.6)$ & $2(1.4)$ & \\
\hline Estimated blood loss (ml), $\mathrm{M} \pm \mathrm{SD}$ & $1.3 \pm 0.8$ & $1.3 \pm 0.9$ & $1.3 \pm 0.8$ & $1.3 \pm 0.8$ & 0.74 \\
\hline Hospital stay (days), $\mathrm{M} \pm \mathrm{SD}$ & $14.7 \pm 16.8$ & $13.7 \pm 14.5$ & $13.2 \pm 8.4$ & $17.5 \pm 24.9^{\mathrm{b}}$ & 0.050 \\
\hline Adjuvant chemotherapy, $\mathrm{n}(\%)$ & $79(16.2)$ & $30(19.4)$ & $26(14.0)$ & $23(15.6)$ & 0.39 \\
\hline 30-day mortality, n (\%) & $15(3.1)$ & $7(4.5)$ & $1(0.5)^{\mathrm{a}}$ & $7(4.8)^{b}$ & 0.039 \\
\hline 90-day mortality, n (\%) & $31(6.4)$ & $19(12.3)$ & $2(1.1)^{\mathrm{a}}$ & $10(6.8)^{b}$ & $<0.001$ \\
\hline Bladder cancer death, $\mathrm{n}(\%)$ & $197(40.4)$ & $74(47.7)$ & $74(39.8)$ & $49(33.3)^{\mathrm{a}}$ & 0.038 \\
\hline Overall death, n (\%) & $363(74.4)$ & $117(75.5)$ & $138(74.2)$ & $108(73.5)$ & 0.92 \\
\hline
\end{tabular}

${ }^{a} \mathrm{p}<0.05$ vs. normal weight. ${ }^{\mathrm{b}} \mathrm{p}<0.05$ vs. overweight. M: mean; SD: standard deviation. 


\begin{tabular}{|c|c|c|c|}
\hline \multicolumn{4}{|c|}{$\begin{array}{l}\text { Table 3. Cox proportional HRs and } 95 \% \text { CIs for the association of body mass index } \\
\text { with 90-day mortality, bladder cancer-specific mortality, and all-cause mortality }\end{array}$} \\
\hline \multirow[b]{2}{*}{ Body mass index $\left(\mathrm{kg} / \mathrm{m}^{2}\right)$} & \multicolumn{3}{|c|}{ Overall $(n=488)$} \\
\hline & $\begin{array}{l}\text { No. of deaths/ } \\
\text { no. of person- } \\
\text { years }\end{array}$ & HR $(95 \%$ CI) & $\mathbf{p}$ \\
\hline \multicolumn{4}{|l|}{ 90-day mortality } \\
\hline Normal weight (18.5-24.9) & $19 / 985$ & Referent & \\
\hline Overweight (25-29.9) & $2 / 1417$ & $0.09(0.02-0.38)$ & 0.001 \\
\hline Obese $(\geq 30)$ & $10 / 1071$ & $0.63(0.29-1.39)$ & 0.25 \\
\hline Per 1-unit increase & & $0.93(0.85-1.02)$ & 0.11 \\
\hline \multicolumn{4}{|l|}{$\begin{array}{l}\text { Bladder cancer-specific } \\
\text { mortality }\end{array}$} \\
\hline Normal weight (18.5-24.9) & $74 / 985$ & Referent & 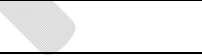 \\
\hline Overweight (25-29.9) & $74 / 1417$ & $0.79(0.57-1.10)$ & 0.16 \\
\hline Obese $(\geq 30)$ & $49 / 1071$ & $0.76(0.52-1.09)$ & 0.13 \\
\hline Per 1-unit increase & & $0.98(0.95-1.01)$ & 0.21 \\
\hline \multicolumn{4}{|l|}{ All-cause mortality } \\
\hline Normal weight (18.5-24.9) & $117 / 985$ & Referent & \\
\hline Overweight $(25-29.9)$ & $138 / 1417$ & $0.84(0.65-1.09)$ & 0.18 \\
\hline Obese $(\geq 30)$ & $108 / 1071$ & $1.00(0.77-1.31)$ & 1.00 \\
\hline Per 1-unit increase & & $1.01(0.98-1.03)$ & 0.70 \\
\hline $\begin{array}{l}\text { All-cause mortality with time- } \\
\text { varying covariate }\end{array}$ & & & $0.020^{*}$ \\
\hline \multicolumn{4}{|l|}{$\leq 63$ months } \\
\hline $\begin{array}{l}\text { Normal weight (18.5- } \\
24.9)\end{array}$ & $88 / 136$ & Referent & \\
\hline Overweight (25-29.9) & $82 / 166$ & $0.66(0.49-0.90)$ & 0.008 \\
\hline Obese $(\geq 30)$ & $72 / 141$ & $0.88(0.64-1.20)$ & 0.42 \\
\hline \multicolumn{4}{|l|}{$>63$ months } \\
\hline $\begin{array}{l}\text { Normal weight }(18.5- \\
24.9)\end{array}$ & $29 / 841$ & Referent & \\
\hline Overweight (25-29.9) & $56 / 1244$ & $1.41(0.89-2.23)$ & 0.14 \\
\hline Obese $(\geq 30)$ & $36 / 920$ & $1.38(0.84-2.28)$ & 0.21 \\
\hline
\end{tabular}

*p value for time-interaction Adjusted for age, sex, cancer subtype, lymphovascular invasion, tumor grade, pathologic $\mathrm{T}$ stage, pathologic $\mathrm{N}$ stage, and adjuvant chemotherapy. HR: hazard ratio; $\mathrm{CI}$ : confidence interval. 